\title{
The ATAF1 transcription factor: At the convergence point of ABA-dependent plant defense against biotic and abiotic stresses
}

\author{
Brigitte Mauch-Mani ${ }^{1}$, Victor Flors ${ }^{2}$ \\ ${ }^{1}$ University of Neuchâtel, Faculty of Science, Department of Biology, Laboratory of Molecular and Cellular Biology, Rue Emile Argand \\ 11, CP 158, CH-2009 Neuchâtel, Switzerland; ' Plant Physiology Section, Department of CAMN, Universitat Jaume I, Spain \\ Cell Research (2009) 19:1322-1323. doi: 10.1038/cr.2009.135; published online 3 December 2009
}

Because of their sessile lifestyle, plants have evolved sophisticated ways of coping with the various biotic and abiotic stresses they can encounter during their life. Their defensive reactions to a given stress have to be rapid and well adapted to the situation. They are the results of tightly coordinated changes at the molecular level involving the contributions of different signaling pathways. Traditionally, two signal transduction pathways have been investigated preferentially for defense against biotic stresses, attributing a stronger involvement in defense against biotrophic or hemibiotrophic organisms to the salicylic acid (SA)-dependent pathway and a major role in defense against necrotrophs to the jasmonic acid/ethylene (JA/ ET)-dependent pathway. In contrast, it is common knowledge that abiotic stress regulation mainly depends on the ABA signaling pathway (for review on the different pathways, see [1]). Recent studies, however, show a role for ABA not only against abiotic stresses but also in defense against pathogens and point to a significant coordination of the plants' responses to the various environmental and biotic stimuli including an important cross-talk between different

$\overline{\text { Correspondence: Brigitte Mauch-Mani }}$ E-mail: brigitte.mauch@unine.ch signaling pathways [2].

NAC (NAM, ATAF, and CUC) transcription factors belong to a gene family specific to plants and play roles in development and stress responses [3]. The first described NAC genes NAM (no apical meristem) and CUC2 (cupshaped cotyledon), as many later ones, are development-related. In Arabidopsis alone, more than a hundred $N A C$ genes have been predicted and many of them have overlapping functions. The members of the ATAF subfamily of $N A C$ domain genes, $A T A F 1$ and $A T A F 2$, were primarily known to negatively regulate responses to drought and wounding and to reduce $P R$ protein expression against some fungi [4, 5], but recent transcription profile data [4] as well as functional studies of $A T A F 1$ and related $N A C$ s show a co-regulated expression by wounding, infection, methyl jasmonate, abscisic acid, hydrogen peroxide, cold, drought, salt and osmotic stresses.

In the non-host plant-pathogen system Blumeria graminis $f$. sp. hordei (Bgh) - Arabidopsis, ATAF1 has been shown to promote penetration resistance [4]. The observed ATAF1-dependent regulation of ABA-responsive genes was clearly correlated to a reduction of ABA levels upon attempted infection of the plants with $B g h$. In atafl mutants, however, such a $B g h$-dependent decrease in ABA levels was not observed.
Wild-type plants also had higher basal levels of ABA than the mutants (atafl) suggesting that the transcriptional regulation of ABA biosynthesis through ATAF1 is stimulus-dependent. Taken together, this shows that the ability of Arabidopsis to restrict penetration by $B g h$ strongly depends on ATAF1-mediated repression of ABA biosynthesis. The obvious hyperinduction, in $B g h$-infected atafl mutants, of ABA-inducible genes, that normally play a role in abiotic stress resistance, points to a similar antagonistic function of ATAF1 as has been described for ERD15 [6]. Thus, ATAF1 is at the crossroad of biotic and abiotic stress pathways and acts as a switch between plant abiotic stress tolerance and defense.

More, although sometimes controversial, evidence regarding the possible roles of ATAF1 has been published lately. atafl mutants have been described to be more drought tolerant [4, 5] as well as less drought tolerant [7]. In these assays, atafl mutants were grown first on agar medium and then transplanted to soil where water was withheld from them after a certain time of growth. In one case [7] atafl mutants recovered much better than wild type plants after re-watering. On the opposite, and using the same Salk insertional mutants, no differences in the recovery rate were found between both wild type 
and mutants, respectively, by Wu et al. [8]. A further controversy can be found after infection with the necrotrophic fungal pathogen Botrytis cinerea. Although there is a consensus, that ATAF1-overexpressing lines are more susceptible to Botrytis infection [8, 9], the expression patterns of defense genes differed in both cases. While Wu et al. [8] describe a down-regulation of $P R 1$ expression after infection compared to wild type, Wang et al. [9] present an up-regulation of $P R 1$ expression. Similarly, $P D F 1.2$ expression goes down in one case [8] and remains stable in the other one [9]. Infection with avirulent Pseudomonas syringae pv tomato avr Rpm 1 and the virulent wild type Pst DC3000 failed to reveal any differences in disease phenotype and severity when comparing wild type and overexpressing lines for ATAF1 [7] but tests with only Pst DC3000 infection of wild type and ATAF1-overexpressing lines yielded a higher disease severity in the overexpressing lines when performed in another lab [8].

At this point, it is not possible to resolve where such discrepancies in the results might stem from. The observations are, however, reminiscent of some controversial reports concerning the role of ABA in disease resistance [2, 6]. Lately, evidence has been pointing into the direction of a strong influence of environmental factors in the modulation of the crosstalk between ABA-signaling, and defined biotic and abiotic stress signaling pathways [10]. Any change resulting in an alteration of ABA homeostasis in plants might therefore punctually destabilize the system and alter the balance of the different pathways involved and it looks like ATAF1 might be prominently involved in this process.

In conclusion, there is increasing evidence that transcription factors integrate abiotic stress tolerance and defenses and most reports show that they act through downstream modulation of responses to $\mathrm{ABA}$.

\section{References}

1 Robert-Seilaniantz A, Navarro L, Bari RJ, et al. Pathological hormone imbalances. Curr Opin Plant Biol 2007; 10:372-379.

2 Mauch-Mani B, Mauch F. The role of abscisic acid in plant-pathogen interactions. Curr Opin in Plant Biol 2005; 8:409-414.

3 Olsen AN, Ernst HA, Leggio LL, et al. NAC transcription factors: structurally distinct, functionally diverse. Trends Plant Sci 2005; 10:79-87.

4 Jensen KM, Hagedorn PH, de Torres-Zabala $\mathrm{M}$, et al. Transcriptional regulation by an NAC (NAM-ATAF1,2-CUC2) transcription factor attenuates $\mathrm{ABA}$ signalling for efficient basal defence towards Blumeria graminisf. sp. hordei in Arabidopsis. Plant $J$ 2008; 56:867880.

5 Delessert C, Kazan K, Wilson IW, et al. The transcription factor ATAF2 represses the expression of pathogenesis-related genes in Arabidopsis. Plant $J$ 2005; 43:745-757.

6 Ton J, Flors V, Mauch-Mani B. The multifaceted role of ABA in disease resistance. Trends Plant Sci 2009; 14:310-317.

7 Lu PL, Chen NZ, An R, et al. A novel drought-inducible gene, $A T A F 1$, encodes a NAC family protein that negatively regulates the expression of stress responsive genes in Arabidopsis. Plant Mol Biol 2007; 63:289-305.

8 Wu Y, Deng Z, Lai J, et al. Dual function of Arabidopsis ATAF1 in abiotic and biotic stress responses. Cell Res 2009; 19:1279-1290.

9 Xiao'e Wang X, Vindhya BM, Basnayake $\mathrm{S}$, et al. The Arabidopsis ATAF1, a NAC transcription factor, is a negative regulator of defense responses against necrotrophic fungal and bacterial pathogens. Mol Plant Microbe Interact 2009; 22:1227-1238.

10 Luna E, Robert J, Flors V, et al. Role of ABA and abiotic factors on PAMPinduced callose deposition. In: Biology of Plant Microbe interactions (Eds. Hani Antoun, et al.) IS-MPMI, St. Paul, Minnesota, USA 2009. 